\title{
Semiconductor-less Photovoltaic Device
}

Fatih B. Atar, ${ }^{1,2}$ Enes Battal, ${ }^{1,2}$ Levent E. Aygun, ${ }^{1,2}$ Bihter Daglar, ${ }^{2}$ Mehmet Bayindir ${ }^{2,3}$ and Ali K. Okyay ${ }^{1,2}$

${ }^{1}$ Department of Electrical and Electronics Engineering, Bilkent University, Ankara 06800, Turkey

${ }^{2}$ UNAM - Institute of Materials Science and Nanotechnology, Bilkent University, Ankara 06800, Turkey

${ }^{3}$ Department of Physics, Bilkent University, Ankara 06800, Turkey

Keywords: Hot electron, photovoltaics, surface plasmons, metal-insulator-metal (MIM)

We demonstrate a novel semiconductor-less photovoltaic device and investigate the plasmonic effects on this device structure. The device is made of metal and dielectric layers and the operation is based on hot carrier collection. We present the use of surface plasmons to improve energy conversion efficiency. The field localization provided by surface plasmons confine the incident light in the metal layer, increasing the optical absorption and hot electron generation rate inside the metal layer. The device consists of two tandem MIM (metalinsulator-metal) junctions. Bottom MIM junction acts as a rectifying diode and top MIM junction is used to excite surface plasmons. The device operation principle as well as the topology will be discussed in detail.

Thermal evaporation and atomic layer deposition (ALD) are used for the deposition of metal and insulator layers, respectively. Au- $\mathrm{HfO}_{2}-\mathrm{Al}$ layers form the bottom MIM junction, and gold nanoparticles (NPs) spin coated on an insulating spacer layer $\left(\mathrm{Al}_{2} \mathrm{O}_{3}\right)$ complete the device structure. Scanning Electron Microscopy (SEM) is used to confirm the randomly distributed 50-nm-diameter Au nanoparticles on the device surface. The design parameters related to the thicknesses of different layers will be discussed in terms of electrical and plasmonic properties of the structure.

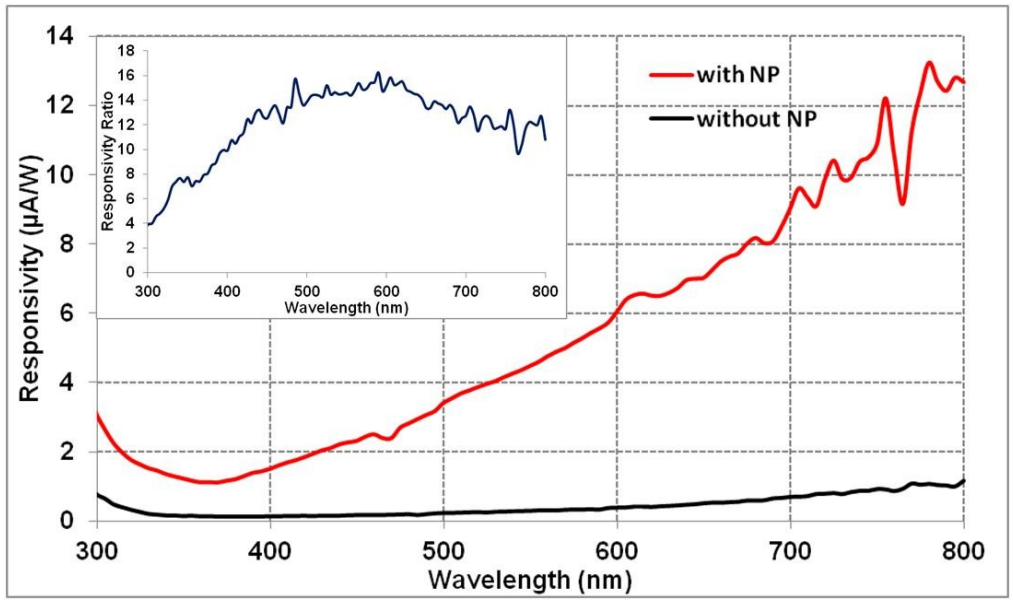

Fig. 1: Measured responsivity of the device before and after nanoparticle spinning. The responsivity ratio of the two measurements is plotted in the inset. The device with nanoparticles shows resonant responsivity enhancement at $600 \mathrm{~nm}$ wavelength. The resonant behaviour of the gold nanoparticles on the device surface is in good agreement with the FDTD simulation results.

As a proof-of-concept demonstration the short circuit current values of the fabricated devices with and without gold nanoparticles were measured under monochromated light. The measurements show clear evidence of the resonant characteristics of Au nanoparticles (Fig 1). Resonant enhancement in the short circuit current is attributed to the excitation of localized 
surface plasmon modes and propagating surface plasmon modes. Finite-difference timedomain (FDTD) simulations were conducted to investigate the surface plasmon modes on the MIM structure.

Significant enhancement in the short circuit current, despite the sparse distribution of gold nanoparticles, clearly shows that the efficiency of MIM photovoltaic devices can be greatly enhanced by surface plasmon excitation. The simple planar MIM structure and use of chemically synthesized nanoparticles for plasmon excitation make this device promising for large area fabrication. We also show that plasmon excitation structures can be introduced without modifying the tunneling (rectifying) MIM junction. This gives additional degrees of freedom to separately optimize the electrical and optical properties of the MIM hot electron photovoltaic device. Further studies can reveal a path for very low cost and sufficiently efficient photovoltaic cells. 